\title{
On a Method of Solving Some Functional Equations for Set-Valued Functions
}

\author{
Justyna Sikorska 1
}

Received: 21 December 2016 / Accepted: 28 November 2017 / Published online: 5 December 2017

(C) The Author(s) 2017. This article is an open access publication

\begin{abstract}
In the first part of the paper we collect and prove several properties of the Hausdorff metric and the Hukuhara difference. They are applied in the next part where a tool for solving several equations for set-valued functions is described.
\end{abstract}

Keywords Set-valued function · Hukuhara difference · Hausdorff distance - Additive function · Quadratic function · Drygas equation · Orthogonally additive equation

Mathematics Subject Classification (2010) 54 C60 - 39B52 · 39B55

\section{Introduction}

Treating the stability problem of functional equations for single-valued functions in several variables we may use several methods. They mostly start with studying the stability of a single variable functional equation. In a general form, we may write such an equation in a form

$$
f(x)=\sum_{i=1}^{n} \alpha_{i} f\left(\varphi_{i}(x)\right) .
$$

It turns out that apart of the results where the above form with one summand on the righthand side is used, for a number of classical equations the following form is applied (see, e.g., [13])

$$
f(x)=\frac{\beta+1}{2 \beta^{2}} f(\beta x)-\frac{\beta-1}{2 \beta^{2}} f(-\beta x) .
$$

Justyna Sikorska

justyna.sikorska@us.edu.pl

1 Institute of Mathematics, University of Silesia, Bankowa 14, PL-40-007 Katowice, Poland 
In what follows we want to show how the set-valued version of (1) helps to find solutions of several equations for multivalued functions in several variables. Some of them have in the background important applications in physics or in economics.

However, before we present results concerning set-valued functional equations, in the second section we introduce several properties of the Hausdorff distance and the Hukuhara difference which are important and interesting in itself (see, e.g., Lemma 2.5 or Lemma 2.6).

In the paper, for a topological vector space $Y$, we denote by $c(Y), c c(Y)$ and $b c l(Y)$ - the family of all nonempty compact, nonempty compact convex and nonempty bounded closed subsets of $Y$, respectively.

\section{Preliminary Results}

In what follows we give some notations and present results which will be used in the sequel. First two results come from Rådström [12] (see also [15] and [14]).

Lemma 2.1 Let $A, B$ and $C$ be sets in a topological vector space. Suppose $B$ is closed and convex, $C$ is bounded, nonempty, and that $A+C \subset B+C$. Then $A \subset B$.

Corollary 2.1 If $A, B$ are closed and convex sets in a topological vector space and $C$ is bounded and nonempty, then $A+C=B+C$ implies $A=B$.

Let $(Y, d)$ be a metric space and let $A, B \in \operatorname{bcl}(Y)$. We define the Hausdorff distance between $A$ and $B$ as follows:

$$
h(A, B):=\max \left\{\sup _{x \in A} d(x, B), \sup _{y \in B} d(A, y)\right\},
$$

where $d(x, B):=\inf \{d(x, y): y \in B\}$. Equivalently (see [2, Section 3.2]),

$$
h(A, B)=\sup _{y \in Y}|d(y, A)-d(y, B)|,
$$

or

$$
h(A, B)=\inf \left\{\varepsilon>0: A \subset B_{\varepsilon} \text { and } B \subset A_{\varepsilon}\right\},
$$

where $A_{\varepsilon}=\cup_{a \in A} K(a, \varepsilon)$ and $K(a, \varepsilon)=\{x \in Y: d(a, x)<\varepsilon\}$. If $d$ is a (translation) invariant metric on a linear space, then $A_{\varepsilon}=A+K(\varepsilon)$, where $K(\varepsilon)$ is an open ball of radius $\varepsilon$ centered at the origin. The space $b c l(Y)$ equipped with the Hausdorff distance is a metric space. Moreover, if $(Y, d)$ is complete, so do $b c l(Y), c(Y)$ and $c c(Y)$, if considered with the Hausdorff metric (see, e.g., [3] or [2]).

In what follows, by the symbol $A_{n} \rightarrow A$ we mean that a sequence of sets $\left(A_{n}\right)_{n \in \mathbb{N}}$ is convergent to $A$ with respect to the Hausdorff metric. For the convenience of the reader, in the next lemma we recall from [14] some properties of this convergence.

Lemma 2.2 Let $(Y, d)$ be a metric linear space. Then

(a) for all $A_{n}, B_{n}, A, B \in \operatorname{bcl}(Y), n \in \mathbb{N}$, if $A_{n} \rightarrow A$ and $B_{n} \rightarrow B$, then $A_{n}+B_{n} \rightarrow$ $A+B$

(b) if $d$ is invariant, then for any $A, B \in \operatorname{bcl}(Y)$ and some $\varepsilon>0$,

$$
h(A, B)<\varepsilon \quad \Longrightarrow \quad(A \subset B+K(\varepsilon) \wedge B \subset A+K(\varepsilon)) \quad \Longrightarrow \quad h(A, B) \leqslant \varepsilon ;
$$

(c) if $d$ is invariant, $A \in \operatorname{bcl}(Y)$ and $\left(\alpha_{n}\right)_{n \in \mathbb{N}}$ is a sequence of real numbers converging to $\alpha \in \mathbb{R}$ then $\alpha_{n} A \rightarrow \alpha A$. 
On account of Lemma 2.2(b), the convergence of a sequence of sets with respect to the Hausdorff metric can equivalently be defined as: $A_{n} \rightarrow A$ if and only if for every neighborhood $V$ of zero in $Y$ (or element of a basis of neighborhoods of zero) there exists $n_{0} \in \mathbb{N}$ such that $A_{n} \subset A+V$ and $A \subset A_{n}+V$ for every $n \geqslant n_{0}$.

While solving functional equations of a single variable we often split functions into their odd and even parts and look first for the solutions of each part separately. In the case of setvalued functions the same methods cannot be used directly (cf., [14]). This is because for the Minkowski difference $A-B=\{a-b: a \in A, b \in B\}$, it is not, in general, true that $(A+B)-B$ equals $A$.

For that reason we will use another difference, introduced by Hukuhara [8], and from now on, it will be the only difference used in $c c(Y)$.

Let $Y$ be a real topological vector space and let $A, B, C \in c c(Y)$. We say that $C$ is the Hukuhara difference of $A$ and $B$, i.e., $C=A-B$ if and only if $B+C=A$. Now it is true that $(A+B)-B=A$ for all $A, B \in c c(Y)$. One can show (on account of Corollary 2.1) that if the Hukuhara difference exists, then it is unique. However, it does not always exist. The problem of the existence of the Hukuhara difference in metric linear spaces is treated in the next lemma (see [8]).

Lemma 2.3 Let $(Y, d)$ be a linear metric space and let $A, B \in c c(Y)$. The Hukuhara difference $A-B$ exists if and only if for every $a \in \partial A$ there exists at least one element $c \in Y$ such that

$$
a \in B+c \subset A \text {. }
$$

In order to show the next property of the Hukuhara difference we still need two lemmas.

Lemma 2.4 Let $(Y, d)$ be a metric linear space and $A_{n}, A \in c(Y), n \in \mathbb{N}$. If $A_{n} \rightarrow A$ then

(i) for every $a \in A$ there exists a sequence $\left(a_{n}\right)_{n \in \mathbb{N}}, a_{n} \in A_{n}$ such that $a_{n} \rightarrow a$;

(ii) for every subsequence $\left(A_{n_{k}}\right)_{k \in \mathbb{N}}$ and $a_{n_{k}} \in A_{n_{k}}$ for $k \in \mathbb{N}$, if $a_{n_{k}} \rightarrow x$ then $x \in A$.

Proof For (i), fix arbitrarily $a \in A$ and let for every $n \in \mathbb{N}, a_{n}$ be an element of $A_{n}$ such that it minimizes the distance between $a$ and $A_{n}$. Then $a_{n} \rightarrow a$.

For (ii), assume that $\left(a_{n_{k}}\right)_{k \in \mathbb{N}}$ with $a_{n_{k}} \in A_{n_{k}}(k \in \mathbb{N})$, converges to some $x \in Y$. Let $\left(x_{n}\right)_{n \in \mathbb{N}}$ be a sequence of elements $x_{n} \in A_{n}$ that minimizes the distance from $x$ to $A_{n}$ $(n \in \mathbb{N})$. Then $d\left(a_{n_{k}}, x\right) \geqslant d\left(x_{n_{k}}, x\right)$, and since $a_{n_{k}} \rightarrow x$ then $x_{n_{k}} \rightarrow x$. For every $k \in \mathbb{N}$ we have

$h\left(A_{n_{k}}, A\right)=\sup _{y \in Y}\left|d\left(y, A_{n_{k}}\right)-d(y, A)\right| \geqslant\left|d\left(x, A_{n_{k}}\right)-d(x, A)\right|=\left|d\left(x, x_{n_{k}}\right)-d(x, A)\right|$.

Tending with $k$ to infinity we obtain $0=d(x, A)$ which yields $x \in A$.

Lemma 2.5 Let $(Y, d)$ be a locally convex linear metric space with an invariant metric and let $A, B, C_{n} \in \operatorname{cc}(Y)$ for all $n \in \mathbb{N}$. If $B+C_{n} \rightarrow A$ then

(i) there exists the Hukuhara difference $A-B=: C$;

(ii) $C_{n} \rightarrow C$.

Proof For (i), fix arbitrarily $a \in \partial A$. On account of Lemma 2.4(i), there exist $x_{n} \in B+C_{n}$, $n \in \mathbb{N}$, such that $x_{n} \rightarrow a$. But $x_{n}=b_{n}+c_{n}$ for some $b_{n} \in B$ and $c_{n} \in C_{n}, n \in \mathbb{N}$. By the compactness of $B$, there exists a convergent subsequence of $\left(b_{n}\right)_{n \in \mathbb{N}}$, say, $b_{n_{k}} \rightarrow b \in B$. 
Hence, $c_{n_{k}} \rightarrow a-b=: c$. We prove that $B+c \subset A$. Fix $d \in B$ and consider $\left(d+c_{n_{k}}\right)_{k \in \mathbb{N}}$. We have $d+c_{n_{k}} \in B+C_{n_{k}}$ for all $k \in \mathbb{N}$ and $d+c_{n_{k}} \rightarrow d+c$. On account of Lemma 2.4(ii), $d+c \in A$. By Lemma 2.3, we get the existence of the Hukuhara difference $A-B$. There exists then a nonempty, compact and convex set $C \subset Y$ such that $A=B+C$.

In order to prove (ii), fix arbitrarily $V$, a neighborhood of zero in $Y$. Since $Y$ is locally convex, there exists a convex neighborhood of zero $U$ such that $\bar{U} \subset V$. Since $B+C_{n} \rightarrow$ $B+C$, there exists $n_{0} \in \mathbb{N}$ such that for all $n \geqslant n_{0}$,

$$
B+C_{n} \subset B+C+U \subset B+C+\bar{U}
$$

and

$$
B+C \subset B+C_{n}+U \subset B+C_{n}+\bar{U} .
$$

Since $B$ is bounded and $C+\bar{U}, C_{n}+\bar{U}$ are closed and convex, on account of Lemma 2.1,

$$
C_{n} \subset C+\bar{U} \subset C+V \text { and } C \subset C_{n}+\bar{U} \subset C_{n}+V,
$$

for all $n \geqslant n_{0}$, which means that $C_{n} \rightarrow C$.

Now we are able to show the following result.

Lemma 2.6 Let $(Y, d)$ be a locally convex linear metric space with an invariant metric. Let $A, A_{n}, B, B_{n} \in \operatorname{cc}(Y)$ be such that $A_{n} \rightarrow A$ and $B_{n} \rightarrow B$. Let, moreover, for each $n \in \mathbb{N}$ there exists the Hukuhara difference $A_{n}-B_{n}$. Then there exists the Hukuhara difference $A-B$ and $A_{n}-B_{n} \rightarrow A-B$.

Proof Denote $C_{n}:=A_{n}-B_{n}, \quad n \in \mathbb{N}$. Obviously, $C_{n} \in \operatorname{cc}(Y), n \in \mathbb{N}$. Let $V$ be a neighborhood of zero in $Y$. There exist a convex neighborhood of zero $W$ such that $\bar{W} \subset V$. Further, there exists a neighborhood $U$ of zero such that $U+U \subset W$. Since $A_{n} \rightarrow A$ and $B_{n} \rightarrow B$, there exists $n_{0} \in \mathbb{N}$ such that for all $n \geqslant n_{0}$,

$$
A_{n} \subset A+U, \quad A \subset A_{n}+U, \quad B_{n} \subset B+U, \quad B \subset B_{n}+U .
$$

Hence,

$$
A_{n}+B \subset A+B_{n}+U+U \subset A+B_{n}+\bar{W}
$$

and

$$
A+B_{n} \subset A_{n}+B+U+U \subset A_{n}+B+\bar{W},
$$

for all $n \geqslant n_{0}$. But $A_{n}=B_{n}+C_{n}$ for all $n \in \mathbb{N}$, so

$$
B_{n}+C_{n}+B \subset A+B_{n}+\bar{W} \text { and } A+B_{n} \subset B_{n}+C_{n}+B+\bar{W},
$$

for all $n \geqslant n_{0}$, and consequently, $B+C_{n} \rightarrow A$.

Remark 2.1 The above lemma generalizes the result from [14, Lemma 2.3], where additionally the completeness of $Y$ was assumed.

\section{A Single Variable Equation}

As told in Introduction, we are going to provide a general method for finding solutions to a number of functional equations. 
Theorem 3.1 Let $\beta$ be a positive integer different from 1 , let $(X,+)$ be a group uniquely divisible by $\beta$, and let $(Y, d)$ be a locally convex linear metric space with an invariant metric. Assume that $F: X \rightarrow \operatorname{cc}(Y)$ satisfies

$$
F(x)+\frac{\beta-1}{2 \beta^{2}} F(-\beta x)=\frac{\beta+1}{2 \beta^{2}} F(\beta x), \quad x \in X .
$$

Then $F$ is of the form $F=a+G$, where $a: X \rightarrow Y$ is an odd function satisfying $a(\beta x)=$ $\beta a(x)$ for all $x \in X$, and $G: X \rightarrow c c(Y)$ is an even set-valued function with $G(\beta x)=$ $\beta^{2} G(x)$ for all $x \in X$. Moreover, such a representation is unique.

Proof By induction and by means of Corollary 2.1, we show that for every $n \in \mathbb{N}$,

$$
F(x)+\frac{\beta^{n}-1}{2 \beta^{2 n}} F\left(-\beta^{n} x\right)=\frac{\beta^{n}+1}{2 \beta^{2 n}} F\left(\beta^{n} x\right), \quad x \in X .
$$

Hence, by the unique divisibility by $\beta$, for every $n \in \mathbb{N}$ there exists the Hukuhara difference $\frac{\beta^{n}+1}{2 \beta^{n}} F(x)-\frac{\beta^{n}-1}{2 \beta^{n}} F(-x)$ and it is equal to $\beta^{n} F\left(\frac{x}{\beta^{n}}\right)$. On account of Lemma 2.6 and Lemma 2.2(d), for every $x \in X$ there exists the Hukuhara difference of the respective limits, that is, $\frac{1}{2} F(x)-\frac{1}{2} F(-x)$.

With $H(x):=\frac{1}{2} F(x)-\frac{1}{2} F(-x)$ and $G(x):=\frac{1}{2} F(x)+\frac{1}{2} F(-x)$ for all $x \in X$, it is easy to see that $F(x)=G(x)+H(x)$ for all $x \in X$. By definition of $H$, for every $x \in X$ we have $\frac{1}{2} F(x)=H(x)+\frac{1}{2} F(-x)$, and also $\frac{1}{2} F(-x)=H(-x)+\frac{1}{2} F(x)$. Consequently,

$$
\frac{1}{2} F(x)=H(x)+H(-x)+\frac{1}{2} F(x), \quad x \in X .
$$

On account of Corollary 2.1,

$$
H(x)+H(-x)=\{0\}, \quad x \in X,
$$

which means that $H$ is single-valued, that is, $H(x)=\{a(x)\}, x \in X$, for some $a: X \rightarrow Y$.

Substituting $F=a+G$ into (3) for a fixed $x \in X$ and then for $-x$, and combining these two equalities we obtain $a(\beta x)=\beta a(x)$ and, consequently, also $G(\beta x)=\beta^{2} G(x)$ for all $x \in X$.

For proving the uniqueness of the representation, let $a_{1}: X \rightarrow Y$, and $G_{1}: X \rightarrow \operatorname{cc}(Y)$ be the second pair of functions satisfying the assertion of the theorem. Then

$$
\begin{gathered}
F(x)=a(x)+G(x)=a_{1}(x)+G_{1}(x), \\
a\left(\beta^{n} x\right)+G\left(\beta^{n} x\right)=a_{1}\left(\beta^{n} x\right)+G_{1}\left(\beta^{n} x\right), \\
\frac{1}{\beta^{n}} a(x)+G(x)=\frac{1}{\beta^{n}} a_{1}(x)+G_{1}(x), \quad n \in \mathbb{N}, x \in X,
\end{gathered}
$$

whence, $G=G_{1}$ and, on account of Corollary 2.1 and (4), $a=a_{1}$.

Remark 3.1 If we assume that $X$ in Theorem 3.1 is a linear space, we may suppose that $\beta$ is any real from $(1, \infty)$.

\section{Applications}

In this section we give some applications of Theorem 3.1 to solving several set-valued functional equations. 
We start with the equation of the form

$$
f(x+y)+f(x-y)=2 f(x)+f(y)+f(-y)
$$

which was introduced in 1987 by Drygas in [4]. The author was looking for characterizations of quasi-inner product spaces, which in turn led to solutions of some problems in statistics and mathematical programming. Equation (5) is now known in the literature as the Drygas equation. For solving the equation for single-valued functions from an abelian group into a uniquely 2-divisible abelian group one may consult, e.g., [5].

In the following theorem we generalize a result proved by W. Smajdor in [17] for functions mapping a linear space into a family of subsets of a Banach space. It is worth underlining that among others we get rid here of the completeness assumption which while solving the equation does not seem to be natural.

Theorem 4.1 If $(X,+)$ is an abelian group uniquely divisible by $2,(Y, d)$ is a locally convex linear metric space with an invariant metric and $F: X \rightarrow c(Y)$ is a solution of

$$
F(x+y)+F(x-y)=2 F(x)+F(y)+F(-y), \quad x, y \in X,
$$

then there exist an additive function $a: X \rightarrow Y$ and a quadratic set-valued function $G: X \rightarrow c c(Y)$ (i.e., satisfying for all $x, y \in X$ condition $G(x+y)+G(x-y)=$ $2 G(x)+2 G(y))$, such that

$$
F(x)=a(x)+G(x), \quad x \in X
$$

and this representation is unique.

Conversely, every multifunction of the form (7), where $a$ is additive and $G$ is quadratic, is a solution to (6).

Proof Since $F(0)=\{0\}$ and $F(x)+F(x)=2 F(x)$ for all $x \in X$, we may assume that $F: X \rightarrow c c(Y)$. With $y=x$ in (6) we have

$$
F(2 x)=3 F(x)+F(-x), \quad x \in X,
$$

which by Corollary 2.1 yields

$$
\frac{3}{8} F(2 x)=\frac{1}{8} F(-2 x)+F(x), \quad x \in X,
$$

and we may apply Theorem 3.1 with $\beta=2$. As a result we obtain $F(x)=a(x)+G(x)$ for all $x \in X$, with an even set-valued function $G: X \rightarrow c c(Y)$ and an odd function $a: X \rightarrow Y$. This together with (6) gives that $G$ is quadratic and $a$ satisfies the Jensen functional equation. And since $a(0)=0$, then $a$ is additive. The rest goes along the same lines as in [17].

The first norm characterization of inner product space was given by Fréchet [6] in 1935. He proved that a normed space $(X,\|\cdot\|)$ is an inner product space if and only if, for all $x, y, z \in X$,

$$
\|x+y+z\|^{2}+\|x\|^{2}+\|y\|^{2}+\|z\|^{2}=\|x+y\|^{2}+\|x+z\|^{2}+\|y+z\|^{2} .
$$

In the same year Jordan and von Neumann [9] gave the celebrated parallelogram law characterization of an inner product space. Since then numerous further conditions, characterizing the inner product spaces among the normed spaces, have been shown. 
If we substitute $\|\cdot\|^{2}=: f$ in (8) we obtain equation that is sometimes named after Fréchet. Solutions $f$ of this equation mapping a commutative group $(G,+)$ into a real linear space $X$ can be found, e.g., in [10]. Considering its set-valued version, that is,

$$
F(x+y+z)+F(x)+F(y)+F(z)=F(x+y)+F(y+z)+F(z+x),
$$

where $F: X \rightarrow c(Y),(X,+)$ is a uniquely 2-divisible abelian group and $(Y, d)$ is a locally convex linear metric space with an invariant metric, we see that $F(0)=\{0\}$ and with $-y$ in the place of $z$ we get the Drygas equation which solutions we already know. On the other hand, it is easy to see that $F=a+G$ with additive $a$ and quadratic $G$ satisfies (9).

In what follows we present a result concerning the general solutions of the orthogonal Cauchy equation, that is,

$$
F(x+y)=F(x)+F(y), \quad x, y \in X, x \perp y .
$$

The single-valued version of (10) has its applications in physics, in the theory of an ideal gas (cf., [1]). In a three-dimensional Euclidean space, by means of (10) we obtain the formula for the distribution law of velocities in an ideal gas at a fixed temperature. There are also applications of (10) in actuarial mathematics in a premium calculation principle (cf., [7]): it was shown, namely, that the variance principle is the only covariance-additive premium principle (for further references concerning (10), see [14]).

The following theorem improves the result from [14, Theorem 2.1]. Our goal was to drop the not natural completeness assumption in the target space. However, in order not to go into notational details we assume the domain to be an inner product space (for general settings of the domain see [14]).

Theorem 4.2 Let $X$ be a real inner product space and $(Y, d)$ be a locally convex metric linear space with translation invariant metric. If $F: X \rightarrow c c(Y)$ satisfies $(10)$, then there exist an additive function $a: X \rightarrow Y$ and a quadratic set-valued function $G: X \rightarrow \operatorname{cc}(Y)$ such that $F=a+G$. Moreover, such a representation is unique.

Proof We apply Theorem 3.1 with $\beta=2$. Due to Lemma 2.6 we do not need the completeness assumption for $Y$.

Now we proceed to the next equation, namely

$9 F\left(\frac{x+y+z}{3}\right)+F(x)+F(y)+F(z)=4\left[F\left(\frac{x+y}{2}\right)+F\left(\frac{y+z}{2}\right)+F\left(\frac{z+x}{2}\right)\right]$.

The form of this equation took its origins from some characterizations of the convexity studied by Popoviciu [11]. The following theorem is an immediate application of Theorem 3.1 (cf. also, [16]).

Theorem 4.3 Let $(X,+)$ be an abelian group uniquely divisible by $3,(Y, d)$ a locally convex linear metric space with an invariant metric and let $F: X \rightarrow c c(Y)$ be a solution of (11). If $F(0)=\{0\}$ or, more general, iffor every $x \in X$ there exists the Hukuhara difference $F(x)-F(0)$ then there exist an additive function $a: X \rightarrow Y$ and a quadratic set-valued function $G: X \rightarrow c c(Y)$ such that

$$
F(x)=a(x)+F(0)+G(x), \quad x \in X
$$

and this representation is unique. 
Conversely, each multifunction of the form $F(x)=a(x)+G(x)+B, x \in X$, where $a: X \rightarrow Y$ is additive, $G: X \rightarrow c c(Y)$ is quadratic and $B \in c c(Y)$, is a solution to (11).

Proof By the assumption, there exists a function $\Phi: X \rightarrow \operatorname{cc}(Y)$ such that $F(x)=\Phi(x)+$ $F(0)$ for all $x \in X$. Surely, $\Phi(0)=\{0\}$ and $\Phi$ satisfies (11). For a fixed $x \in X$ we substitute $3 x, 3 x,-3 x$ into (11) in the place of $x, y, z$, respectively. As a consequence, we obtain

$$
9 \Phi(x)+\Phi(-3 x)=2 \Phi(3 x), \quad x \in X .
$$

Theorem 3.1 applied with $\beta=3$ yields the desired assertion. The converse is obvious.

The last equation that we are going to consider in the present paper is

$$
F(x+n y)+n F(x-y)=F(x-n y)+n F(x+y), \quad x, y \in X,
$$

where $n$ is a positive integer greater than one.

Theorem 4.4 Let $(X,+)$ be an abelian group uniquely divisible by 2 and $n,(Y, d)$ a locally convex linear metric space with an invariant metric and let $F: X \rightarrow c c(Y)$ be a solution of (13). If for every $x \in X$ there exists the Hukuhara difference $F(x)-F(0)$, then there exist an additive function $a: X \rightarrow Y$ and a quadratic set-valued function $G: X \rightarrow c c(Y)$ such that $F$ is of the form (12). Moreover, such a representation is unique.

Conversely, each multifunction of the form $F(x)=a(x)+G(x)+B, x \in X$, where $a: X \rightarrow Y$ is additive, $G: X \rightarrow c c(Y)$ is quadratic and $B \in c c(Y)$, is a solution to (13).

Proof Similarly as in the proof of the previous theorem, there exists a function $\Phi: X \rightarrow$ $c c(Y)$ such that $F(x)=\Phi(x)+F(0)$ for all $x \in X, \Phi(0)=\{0\}$ and $\Phi$ satisfies (13). Take $x \in X$ and substitute $y:=x$ in (13) (with $F:=\Phi$ ), and then take $n x,-x$ in the place of $x, y$ in (13), respectively. We get the following two equalities

$$
\begin{gathered}
\Phi(x+n x)=\Phi(x-n x)+n \Phi(2 x), \\
\Phi(2 n x)+n \Phi(n x-x)=n \Phi(n x+x) .
\end{gathered}
$$

By use of Corollary 2.1, we obtain

$$
\Phi(2 n x)+n \Phi((n-1) x)=n \Phi((1-n) x)+n^{2} \Phi(2 x), \quad x \in X .
$$

Change $x$ for $-x$, add the two equalities side by side and use the unique 2-divisibility in order to get

$$
\Phi(n x)+\Phi(-n x)=n^{2} \Phi(-x)+n^{2} \Phi(-x), \quad x \in X .
$$

By (13) with $x:=0$ we have

$$
\Phi(n x)+n \Phi(-x)=\Phi(-n x)+n \Phi(x), \quad x \in X .
$$

Consequently, from (14) and (15) we obtain (3), and by Theorem 3.1 we get the desired assertion.

Remark 4.1 If we suppose (13) with $n=2$ then the induction procedure shows that (13) is valid for all positive integers $n$.

Remark 4.2 If we omit the assumption about the existence of suitable Hukuhara differences in Theorem 4.4, we get

$$
F(n x)+F(-n x)+2\left(n^{2}-1\right) F(0)=n^{2} F(-x)+n^{2} F(-x), \quad x \in X .
$$


By (13) with $x:=0$ we have

$$
F(n x)+n F(-x)=F(-n x)+n F(x), \quad x \in X
$$

instead of (14) and (15), which yields

$$
\frac{1}{n^{2}} F(n x)+\frac{n^{2}-1}{n^{2}} F(0)=\frac{n^{2}+n}{2 n^{2}} F(x)+\frac{n^{2}-n}{2 n^{2}} F(-x) .
$$

On account of Lemma 2.6 for every $x \in X$ there exists the Hukuhara difference $\frac{1}{2}[F(x)+$ $F(-x)]-F(0)$. This means that in the case of an even function $F$ the assumption of the existence of the Hukuhara difference is redundant.

We terminate the paper with the problem stemming from the last two applications of Theorem 3.1.

Problem 4.1 Do the assumptions about the existence of the Hukuhara difference or about the evenness of function $F$ in Theorems 4.3 and 4.4 (see also Remark 4.2) necessary? That is, are all solutions to (11) and (13) of the form $a+G+B$ with additive $a: X \rightarrow Y$, quadratic $G: X \rightarrow c c(Y)$ and $B \in c c(Y)$ ?

Acknowledgments The author is grateful to the Editor and the reviewers for their valuable comments and remarks which helped to improve the final version of the paper.

Open Access This article is distributed under the terms of the Creative Commons Attribution 4.0 International License (http://creativecommons.org/licenses/by/4.0/), which permits unrestricted use, distribution, and reproduction in any medium, provided you give appropriate credit to the original author(s) and the source, provide a link to the Creative Commons license, and indicate if changes were made.

\section{References}

1. Aczél, J., Dhombres, J.: Functional equations in several variables. Encyclopedia Math. Appl. Vol. 31. Cambridge University Press, Cambridge (1989)

2. Beer, G.: Topologies on closed and closed convex sets. Mathematics and its Applications, vol. 268. Kluwer Academic Publishers Group, Dordrecht (1993)

3. Castaing, C., Valadier, M.: Convex analysis and measurable multifunctions. Springer, Berlin-HeidelbergNew York (1977)

4. Drygas, H.: Quasi-inner products and their applications. In: Gupta, A.K. (ed.) Advances in Multivariate Statistical Analysis, pp. 13-30. D. Reidel Publishing Co., Dordrecht (1987)

5. Ebanks, B.R., Sahoo, P.K.: A common generalization of functional equations characterizing normed and quasi-inner product spaces. Canad. Math. Bull 35, 321-327 (1992)

6. Fréchet, M.: Sur la définition axiomatique d'une classe d'espaces vectoriels distanciés applicables vectoriellement sur l'espace de Hilbert. Ann. of Math. (2) 36, 705-718 (1935)

7. Heijnen, B., Goovaerts, M.J.: Additivity and premium calculation principles. Blätter der Deutschen Gesellschaft für Versich. Math 17, 217-223 (1986)

8. Hukuhara, M.: Intégration des applications measurables dont la valeur est un compact convexe. Funkcial. Ekvac. 10, 205-223 (1967)

9. Jordan, P., von Neumann, J.: On inner products in linear, metric spaces. Ann. of Math. (2) 36, 719-723 (1935)

10. Kannappan, P.: Functional Equations and Inequalities with Applications. Springer Monographs in Mathematics. Springer, New York (2009)

11. Popoviciu, T.: Sur certaines inégalités qui caractérisent les fonctions convexes. An. Şti. Univ. "Al. I. Cuza" Iaşi Secţ. I a Mat. (N.S.) 11B, 155-164 (1965)

12. Rådström, H.: An embedding theorem for spaces of convex set. Proc. Amer. Math. Soc. 3, 165-169 (1952) 
13. Sikorska, J.: On a direct method for proving the Hyers-Ulam stability of functional equations. J. Math. Anal. Appl. 372, 99-109 (2010)

14. Sikorska, J.: Set-valued orthogonal additivity. Set-Valued Var. Anal. 23, 547-557 (2015)

15. Smajdor, W.: Subadditive and subquadratic set-valued functions. Prace Nauk. Uniw. Śląsk. 889, Katowice (1987)

16. Smajdor, W.: On a Jensen type functional equation. J. Appl. Anal. 13, 19-31 (2007)

17. Smajdor, W.: On set-valued solutions of a functional equation of Drygas. Aequationes Math. 77, 89-97 (2009) 